\title{
Individualized Ablation of Hepatocellular Carcinoma: Tailored Approaches across the Phenotype Spectrum
}

\author{
Zlatko Devcic, MD ${ }^{1}$ Mohamed Elboraey, $\mathrm{MD}^{1} \quad$ Lucas Vidal, $\mathrm{MD}^{3}$ Kabir Mody, MD \\ Denise Harnois, DO $^{3}$ Tushar Patel, MBChB ${ }^{3}$ Beau B. Toskich, MD ${ }^{1}$
}

${ }^{1}$ Division of Interventional Radiology, Mayo Clinic Florida,
Jacksonville, Florida
${ }^{2}$ Division of Oncology, Mayo Clinic Florida, Jacksonville, Florida
${ }^{3}$ Department of Transplantation, Mayo Clinic Florida, Jacksonville, Florida

Address for correspondence Zlatko Devcic, MD, Division of Interventional Radiology, Mayo Clinic Florida, Jacksonville, FL 32224 (e-mail: devcic.zlatko@mayo.edu).

Semin Intervent Radiol 2019;36:287-297

\begin{abstract}
Keywords

- hepatocellular carcinoma

- locoregional therapy

- ablation

Ablation is now recommended by international guidelines for the definitive treatment of hepatocellular carcinoma (HCC). Extensive clinical studies have demonstrated outcomes comparable to surgical resection with shorter hospital stays, decreased costs, and improved quality of life. Successful ablation requires complete treatment of both tumor and margin while preserving critical adjacent structures. HCC exhibits highly variable presentations in both anatomic involvement and biology which have significant implications on choice of ablative therapy. There are now abundant ablation modalities and adjunctive techniques which can be used to individualize ablation and maximize curative results. This article provides a patient-centered summary of approaches to HCC ablation in the context of patient performance, hepatic reserve, tumor phenotype and biology, intra- and extrahepatic anatomy, and ablation technology.
\end{abstract}

Ablation is now endorsed by international professional societal guidelines, including those of the American Association for the Study of Liver Disease ${ }^{1}$ and the European Association for the Study of the Liver, ${ }^{2}$ and it has been incorporated within the Barcelona Clinic Liver Cancer group schema, ${ }^{3}$ for the definitive treatment of hepatocellular carcinoma (HCC). Randomized controlled trials have demonstrated no difference in overall survival (OS) or recurrencefree survival (RFS) between ablation and surgical resection for small tumors ${ }^{4,5}$ with the added benefits of comparatively shorter hospital stays, ${ }^{6}$ reduced 30 -day admissions, ${ }^{6}$ decreased cost, ${ }^{7}$ and improved quality of life. ${ }^{8}$ Recurrence rates after resection can be as high as $75 \% 5$ years after surgery ${ }^{9-11}$ and $8 \%$ within the first year of transplantation. ${ }^{12}$ These patients may not be candidates for additional surgery but may benefit from ablation of low-volume intrahepatic recurrence.

High-quality ablation requires appropriate patient selection and must consider overall oncologic intent, staging the extent of disease, and estimation of liver reserve after therapy. All treatments should maximize pathologic necrosis of the targeted lesion and the at-risk tissue while preserving critical intra- and extrahepatic anatomy. Many curative stage tumors presenting in unfavorable anatomic locations for thermal ablation may be relegated to palliative therapies such as transarterial chemoembolization (TACE) or systemic therapy. These "orphaned" tumors are generally underrecognized in the literature due to a lack of intention-to-treat data in most ablation studies. Emerging ablation modalities and adjunctive techniques offer an opportunity to individualize the therapeutic approach based on tumor presentation. These choices may be particularly beneficial in patients who are not conventional candidates for thermal ablation. This article provides a tailored approach to definitive HCC ablation in the context of patient health and hepatic reserve, tumor phenotype and biology, intra- and extrahepatic anatomy, and ablation technology.

\section{Patient Health and Hepatic Substrate}

Given the frequent concomitant hazard of liver disease, ablation must be performed in patients with an adequate
Issue Theme Ablative Therapy: Part 1; Guest Editor, Beau B. Toskich, MD
Copyright (C) 2019 by Thieme Medical Publishers, Inc., 333 Seventh Avenue, New York, NY 10001, USA. Tel: +1(212) 584-4662.
DOI https://doi.org/ 10.1055/s-0039-1698755. ISSN 0739-9529. 
life expectancy to benefit from treatment. Performance status is commonly assessed with the Eastern Cooperative Oncology Group (ECOG) or Karnofsky scales. Multiple studies have demonstrated that the ECOG is inversely proportional with survival rates in patients treated with radiofrequency ablation (RFA), ${ }^{13}$ stereotactic body radiation therapy (SBRT), ${ }^{14}$ radioembolization (TARE), ${ }^{15}$ and TACE. ${ }^{16}$ Appropriate ablation candidates typically have an ECOG score $\leq 1$ in which patients demonstrate minimal physical restriction. ${ }^{17}$ Higher ECOG scores $(\geq 2)$ may be acceptable when patients are treated as part of a liver transplantation effort, but are subject to increased toxicity. Furthermore, less toxic therapies may be considered in patients with limited natural life expectancy, at the expense of efficacy, in select circumstances. $^{18}$

Patients undergoing ablative therapy should have adequate volume and functional capacity to tolerate devitalization of the lesion and margin. The total liver function which sustains the patient, or hepatic substrate, is frequently a subjective impression based on summation of factors but can be quantified to some degree using clinical calculators. The albumin-bilirubin grade, Child-Pugh score, and Model for End Stage Liver Disease (MELD) score have all demonstrated useful correlations with survival after locoregional therapy. ${ }^{16,19-23}$ Prognostic models which take into consideration both patient and tumor factors have been recently developed for ablation and may help with clinical decision making. ${ }^{24}$ Ultimately, these instruments should be used to better define the risks of a treatment plan to both the patient and care team.

In addition to objective signs of liver function, the operator should be aware of more inconspicuous factors that may increase the probability of adverse events such as prior external beam radiotherapy, previous systemic therapy, or extrahepatic comorbidities such as diabetes. ${ }^{25}$ Bacterial colonization of bile ducts after previous enterobiliary manipulation has been associated with higher rates of abscess formation after thermal ablation. ${ }^{26}$ With proper preprocedural investigation, many of the complications associated with these factors can be avoided.

\section{Endpoints of High-Quality Ablation}

The intraprocedural objective of high-quality ablation is complete coverage of the lesion and margin with preservation of critical structures. Ablation margins for the treatment of HCC were initially adopted from the surgical literature, which ranged from 5 to $10 \mathrm{~mm}$, and have since been validated with multiple studies. ${ }^{27,28}$ While some authors support a margin of $3 \mathrm{~mm}$ for ablation candidacy, ${ }^{29}$ margins of less than $5 \mathrm{~mm}$ have shown higher rates of local tumor progression (LTP) $)^{30-32}$ and distant intrahepatic recurrence. ${ }^{33}$ Similarly, margins greater than $5 \mathrm{~mm}$ have demonstrated decreased rates of LTP, out-offield progression, and RFS for solitary $\mathrm{HCC} \leq 5 \mathrm{~cm} .{ }^{34,35}$ Margins should be based on imaging predictors of poor biology, such as microvascular invasion (MVI), as resection data have demonstrated margins less than $1 \mathrm{~cm}$ in the setting of MVI result in a twofold increase in both recurrence and mortality. ${ }^{36,37}$ Most studies evaluating HCC treatment margins do not stratify recommendations based on tumor biology and this is a shortcoming of most guidelines.

Interestingly, there are data to suggest that suboptimal ablation has the ability to promote unfavorable biology and encourage nodal recurrence after transplantation. ${ }^{38,39}$ Animal models have demonstrated that more aggressive HCC phenotypes develop through sublethal heat induced activation of extracellular matrix collagen $\mathrm{I}^{40}$ and induction of an epithelial-mesenchymal transition, ${ }^{41}$ while metastatic potential may increase through thermal promotion of vascular endothelial growth factor pathways. ${ }^{42}$ Achieving a complete pathologic response prior to transplantation has shown superior posttransplant 5-year recurrence-free survival rates compared with incomplete responses. ${ }^{43}$ Overall, the combined surgical and locoregional experience suggests that a $5 \mathrm{~mm}$ margin is appropriate for lesions that are welldefined, demonstrate favorable biology, and measure less than $3 \mathrm{~cm}$ and margins of $1 \mathrm{~cm}$ or greater may be required for larger or more aggressive tumors (-Fig. $\mathbf{1}$ ).

Given the potential for harm associated with suboptimal treatment, ablation programs should invest in continuous medical education and quality control efforts to approximate
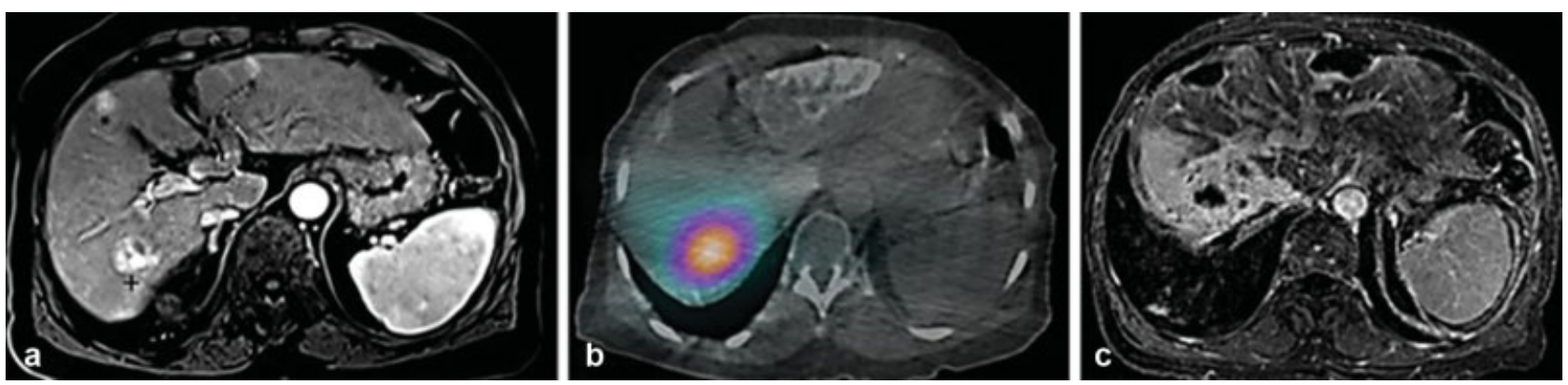

Fig. 1 (a) Contrast-enhanced magnetic resonance imaging (MRI) demonstrates two well-rounded, hypervascular, non-aggressive-appearing hepatocellular carcinomas (HCCs) anteriorly $\left(^{*}\right)$ in addition to a large posterior $\mathrm{HCC}(+)$ with aggressive features including non-smooth margins, irregular rim-like arterial enhancement, and capsular disruption. The posterior lesion required larger ablation margins to cover the tumor, at-risk margin, and adjacent satellite nodules. (b) Bremsstrahlung single-photon emission computed tomography/computed tomography after ablative radioembolization of the posterior lesion demonstrates activity within the targeted angiosomes covering the entire tumor, satellite lesions, and at least a 1-cm at-risk margin. (c) Contrast-enhanced MRI 3 months after treatment demonstrates a complete response to all three HCCs. There are microwave ablation cavities replacing the anterior low-risk lesions with at least a 5 - to 10-mm margin and evolving ablative radioembolization changes to the large posterior aggressive HCC with at least a 1-cm ablative margin. There was no change in baseline liver function after treatment. 
the results of experienced high-volume centers. To assist in the advancement of ablation outcomes, interventional radiology is now a separate medical specialty where residents are given specific training in interventional oncology as part of their board certification.

\section{Tumor Biology}

HCC biology is remarkably heterogeneous and is driven by a complex interplay between molecular subgrouping, genetic alterations, and other oncologic pathways. ${ }^{44}$ Transcriptomebased imaging research suggests that $\mathrm{T}$ stage alone may insufficiently predict tumor biology. ${ }^{45}$ Imaging predictors of aggressive biology include irregular peripheral morphology and peritumoral enhancement, pseudocapsule disruption, satellite formation, and peritumoral hypointensity on hepatobiliary phase imaging. ${ }^{46,47}$ Lower apparent diffusion coefficient values, obtained with $b$-value 0 to 500 second/ $\mathrm{mm}^{2}$, may indicate MVI in small $\mathrm{HCCs} \leq 2 \mathrm{~cm} .{ }^{48}$ Poorly differentiated HCC tends to present with arterial phase hypointensity and $\mathrm{T} 2$ sequence hyperintensity per magnetic resonance imaging. ${ }^{46}$ More established predictors related to aggressive biology include size, ${ }^{49}$ biliary ${ }^{50}$ and portal system involvement, ${ }^{51,52}$ and a doubling time less than 2 months. ${ }^{53}$ The presence of fat is associated with lower rates of MVI. ${ }^{54}$ Developments in radiomic and texture analysis have demonstrated clinical applicability in HCC and may have a future role in tumor characterization. ${ }^{55,56}$

Although $\alpha$-fetoprotein (AFP) is the most practical and widely adopted tumor marker for HCC in clinical practice, it has well known limitations in both sensitivity and specificity where up to $40 \%$ of patients are nonproducers. ${ }^{57,58}$ AFP levels as low as $20 \mathrm{ng} / \mathrm{mL}$ have been associated with MVI, poor differentiation, and tumor recurrence compared with nonAFP-producing tumors, ${ }^{59}$ while levels greater than 1,000 may compromise transplant candidacy. ${ }^{60}$ Elevations in carbohydrate antigen 19-9 in addition to AFP suggest the diagnosis of the biphenotypic hepatocholangiocarcinoma (HCC-CCA). ${ }^{61}$ HCC-CCA tumors are more aggressive, have imaging features anywhere along the HCC or cholangiocarcinoma spectrum, and survival is similar to or worse than intrahepatic cholangiocarcinoma, even in the posttransplant setting. ${ }^{61-63}$

Immunologic ratios such as the serum neutrophil-tolymphocyte ratio and platelet-to-lymphocyte ratio have been associated with lower OS and RFS after RFA of HCC within Milan criteria. ${ }^{64}$ Des-gamma-carboxyprothrombin has been used to predict survival and recurrence after RFA $^{65}$ and the hepatitis $B$ viral load has been shown to correlate with local recurrence after RFA. ${ }^{66}$ Less commonly used biomarkers include AFP-L3, glypican-3, cytokeratin 19, Golgi protein 73, Midkine, osteopontin, squamous cell carcinoma antigen, annexin A2, and lens culinaris agglutinin. ${ }^{67,68}$ Sampling of circulating microRNAs and cell-free DNA in conjunction with clinical presentation may increase the accuracy of HCC diagnosis. ${ }^{67}$ The discovery of biomarkers remains an active area of HCC research where future applications may include early treatment reallocation and incorporation into societal guidelines.

\section{Ablation Modality}

\section{Thermal}

Thermal ablation continues to have the largest published experience of all ablation technologies and RFA carries international recommendations for definitive treatment of $\mathrm{HCC} \leq 3 \mathrm{~cm}$ with favorable anatomy. ${ }^{3}$ These outcomes have been confirmed with post-RFA explant analysis demonstrating HCC complete pathologic necrosis rates ranging from 55 to $100 \%{ }^{69,70}$ Microwave ablation (MWA) has theoretical advantages over RFA by offering increased power, the ability to perform larger ablations, less susceptibility to tissue charring, potentially overcoming the heat sink effect, and decreasing procedure time. While a meta-analysis demonstrated superior tumor control in favor of MWA over RFA, a subsequent randomized controlled trial did not identify a difference in outcomes between the two. ${ }^{71,72}$

Cryoablation is of use in high-risk anatomy due to its ability to actively monitor intraprocedural ice formation (-Fig. 2). While animal models demonstrate the $-20^{\circ} \mathrm{C}$ isotherm is $1 \mathrm{~mm}$ from the edge of the visualized ice ball, ${ }^{73}$ lethal isotherms are highly variable in clinical
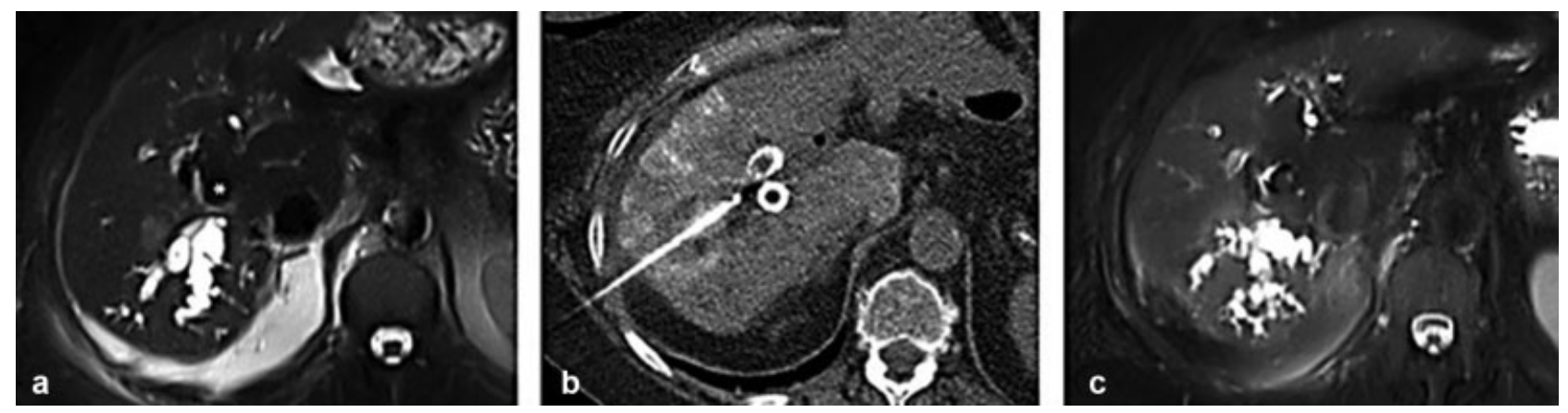

Fig. 2 (a) T2-weighted magnetic resonance imaging (MRI) demonstrates a 1.6-cm hepatocellular carcinoma (HCC) with poor arterial conduit and nearby biliary dilatation in which irreversible electroporation could not be performed due to adjacent parallel transjugular intrahepatic portosystemic shunts (TIPS) (*). (b) Two cryoablation probes were placed through the cranial and caudal aspect of the mass with adequate intraprocedural ice formation encompassing the HCC and margin. (c) T2-weighted MRI image along the same plane demonstrates scaring of the treated region without residual disease. There was no evidence of injury to the bile ducts or the TIPS. 
practice. Cryoablation can generate larger ablations than RFA with reports of improved outcomes for HCC greater than $3 \mathrm{~cm},{ }^{74}$ although larger cryoablations have been loosely associated with rare posttreatment systemic inflammatory reactions termed "cryoreaction" and "cryoshock."75 Cryoablation may be limited by longer procedure times and a conceptual increased susceptibility to the heat sink effect. Laser ablation, which has the unique property of delivering energy via 21-gauge needles, has shown comparable results to RFA for small HCC, but has limited availability and few centers have developed expertise compared with other thermal modalities. ${ }^{76}$

\section{Nonthermal}

Controlled tissue destruction can be achieved via other technologies that do not rely on the alteration of tissue temperature for efficacy. Irreversible electroporation (IRE) generates tumor lethality via the induction of nanoscale cellular lipid bilayer perforations that result in eventual apoptosis rather than coagulative necrosis. This provides a theoretical advantage with high-risk anatomy via the preservation of extracellular matrix in adjacent tissues and being independent of heat-related effects (-Fig. 3). The disadvantages of IRE include the requirement for general anesthesia and meticulous parallel probe placement. IRE has demonstrated promising results with complete response rates between 77 and $92 \%$ for HCCs in challenging locations not amenable to thermal modalities. ${ }^{77}$ A recent explant correlation study demonstrated $83 \%$ of IRE-treated lesions had complete pathologic necrosis. ${ }^{78}$

\section{Chemical}

Percutaneous ethanol injection (PEI) was one of the initially adopted locoregional modalities for HCC. It relies on the caustic properties of ethanol which generate ablative effects via protein denaturation, cellular dehydration, and vascular
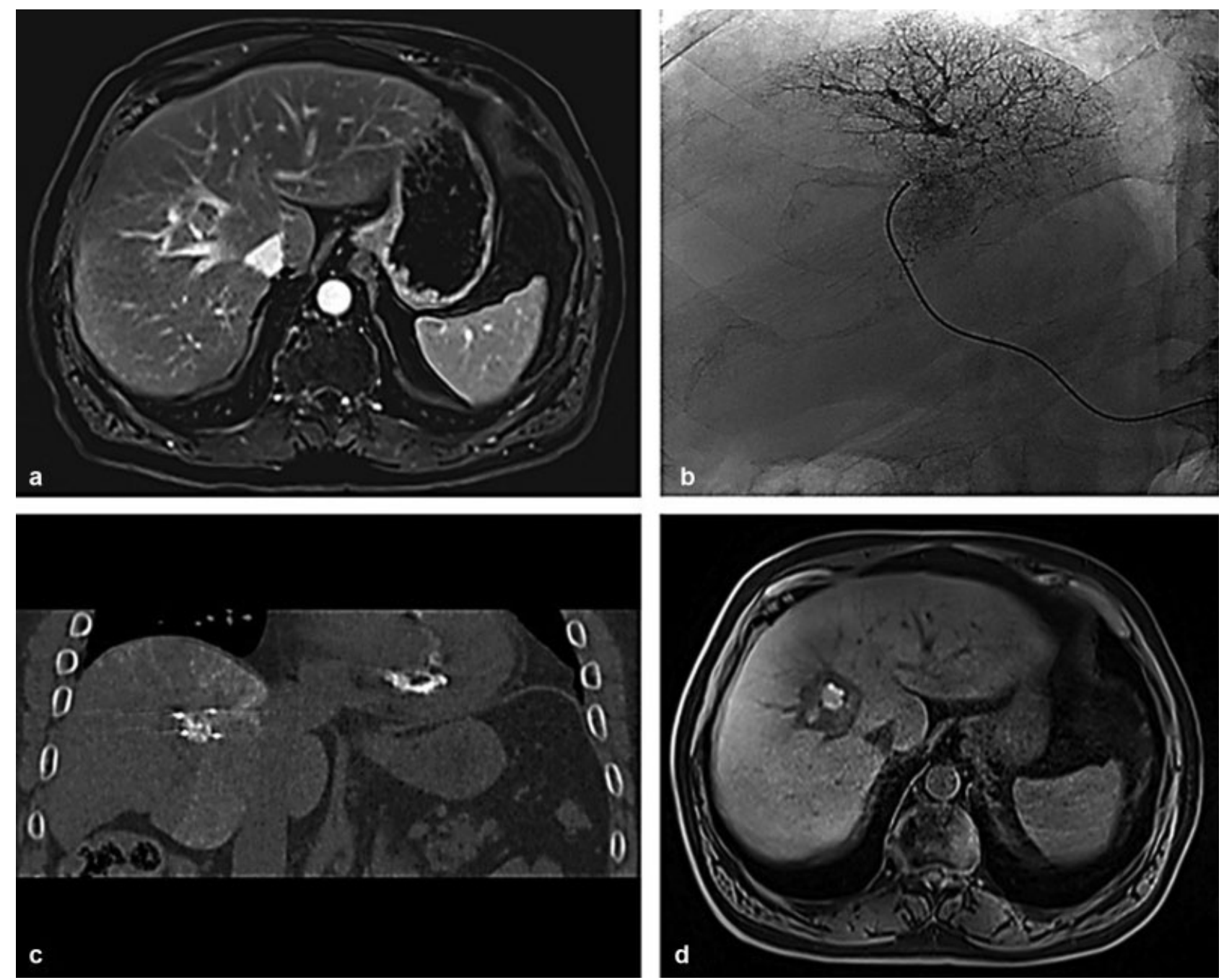

Fig. 3 (a) Contrast-enhanced magnetic resonance imaging (MRI) demonstrates a well-rounded hilar hepatocellular carcinoma adjacent to the central bile ducts and the portal vein which could not be safely and effectively treated with thermal ablation. (b) Radioembolization could not be utilized due to an inadequate microvascular conduit, watershed arterial supply, and nontarget preferential flow. The lesion was stained with lipiodol to improve visualization for subsequent irreversible electroporation (IRE). (c) Intraprocedural noncontrast computed tomography demonstrates placement of four parallel IRE probes bracketing the lesion. (d) Noncontrast MRI 3 months after treatment demonstrates complete response with hemorrhage in the center of the lesion and an adequate margin of ablated tissue. Contrast-enhanced images (not shown) demonstrated no evidence of enhancement and there were no complications. 
thrombosis. Ethanol can be safely injected next to critical structures such as bile ducts and bowel. While it is useful in treating small tumors adjacent to high-risk structures, outcomes are inferior with larger tumors and adequate chemical diffusion within a lesion can be highly variable. ${ }^{79-81}$ Reported rates of PEI complete pathologic necrosis range from $30 \%^{82}$ to $64.3 \%^{83}$ in tumors generally smaller than $3 \mathrm{~cm}$.

Chemical ablation can also be performed via transarterial infusion of ethanol (TAE) with wedged embolization in a 1:2 or 1:3 (alcohol:lipiodol) ratio via the complete saturation of arterioles, sinusoids, peribiliary plexus, and portal venules. Candidacy for TAE relies upon the presence of a favorable arterial supply as a therapeutic conduit. A large study evaluating TAE for both small and large HCCs showed a complete response rate of $69.1 \%$ with a median time to progression (TTP) of 9.1 months, and a progression-free survival (PFS) of 8.4 months. ${ }^{84}$ Compared with TACE, TAE has demonstrated improved TTP and PFS (34.6 vs. 26.1 and 14.8 vs. 9.3 months, respectively) and increased complete response rates at 3, 6, and 12 months. ${ }^{85}$ A meta-analysis of 19 RCTs demonstrated TACE with PEI had higher local tumor control and survival rates than PEI alone. ${ }^{86}$ As such, the addition of ethanol therapy to thermal ablation could improve outcomes where anatomic constraints would have prevented solitary treatment with either modality. ${ }^{87}$

\section{Radiation}

Radioembolization has demonstrated its ability to serve as an ablative therapy in segmental administrations where uniform dose exceeds $190 \mathrm{~Gy}$, commonly referred to as radiation segmentectomy. ${ }^{88}$ There are single-center, longterm, five-year survival data that have shown comparability of radiation segmentectomy to established curative standards. ${ }^{89}$ Radioembolization can safely ablate large volumes (-Fig. 4), including neoadjuvant lobar treatments, in challenging locations while having minimal toxicity on adjacent structures. ${ }^{90,91}$ Radioembolization relies on suitable arterial tumor conduit and adequate margin within the targeted angiosome. Systemic therapies which alter tumor vascularity (e.g., bevacizumab or sorafenib) may affect treatment outcomes. Modulation of suboptimal angiosomes using reversible intra-arterial occlusion has been reported. ${ }^{92}$ Hepatic explant evaluation has demonstrated more than $50 \%$ complete pathologic necrosis ( $>90 \%$ in all treated patients) in solitary tumors $\leq 5 \mathrm{~cm}$ treated with radioembolization which were not amenable to RFA. ${ }^{15}$

When percutaneous or transarterial ablative therapy is not an option, SBRT with either photon or proton radiation has shown 1-year local control rates of $90.9 \%$ and a PFS of 82.7, 58.3, and $36.4 \%$ at 1,3 , and 5 years for $\mathrm{HCC} \leq 5 \mathrm{~cm},{ }^{93}$ respectively. Complete pathologic necrosis rates range from $13 \%{ }^{94}$ to $28 \%{ }^{95}$ for tumors up to $4.5 \mathrm{~cm}$ in patients undergoing bridge to transplantation.

\section{High-Intensity Focused Ultrasound}

High-intensity focused ultrasound (HIFU) is an emerging modality that generates high-energy external compression waves as a means of tissue ablation. Compared with RFA, HIFU has demonstrated similar complete response rates for small recurrent HCC with a mean size of 1.7 to $1.8 \mathrm{~cm}$ without a significant difference in disease-free survival or OS. ${ }^{96}$ It has also demonstrated similar complete response rates (87.2 vs. 94.4\%) and comparable 1- and 3-year OS for tumors less than $3 \mathrm{~cm}$ compared with RFA. ${ }^{97}$ Utilization has been limited due to long procedure times and the limitations of acoustic windows to access targeted tumors.

\section{Anatomical Considerations}

\section{Intrahepatic Anatomy}

The anatomic presentation of HCC has major implications in its ability to undergo successful ablation. Due to the extreme effects of thermal-induced coagulative necrosis, tumors located near the central portal triad have demonstrated higher rates of incomplete coverage after thermal ablation, ${ }^{98,99}$ an increased risk of intrasegmental recurrence, ${ }^{100}$ higher rates of biliary injury, ${ }^{101}$ and increased mortality. ${ }^{102}$ While experienced users may have the ability to engage centrally located tumors in addition to using adjunctive techniques such as bile duct cooling, ${ }^{103}$ effective treatment can still be performed by utilizing nonthermal modalities such as PEI, IRE, ${ }^{104-106}$ and radioembolization. Cryoablation has demonstrated reduced damage to central bile ducts in some reports, but caution should still be exerted. ${ }^{107}$
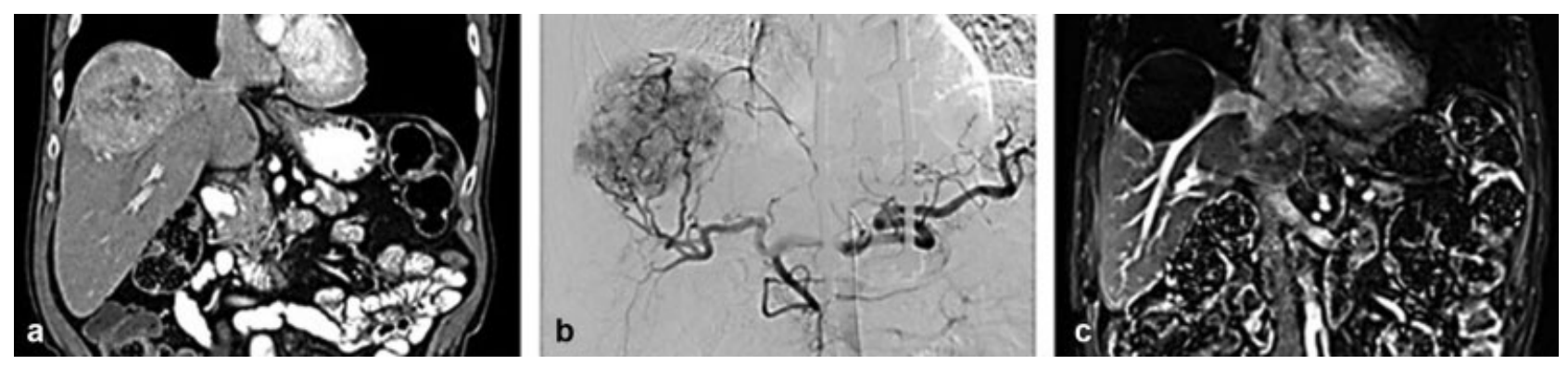

Fig. 4 (a) Contrast-enhanced computed tomography demonstrates a systemic therapy refractory $10 \mathrm{~cm}$ hepatocellular carcinoma at the dome of the liver in a patient who was not a surgical candidate due to a spine metastasis. Treatment of such a large tumor with thermal ablation would have been potentially challenging and toxic in a patient with stage IV disease. (b) Mapping angiography demonstrated a favorable arterial conduit and the patient was subsequently treated with segmental ablative radioembolization. (c) Dynamic contrast-enhanced subtraction magnetic resonance imaging 1 year after treatment continues to demonstrate a modified Response Evaluation Criteria in Solid Tumors complete response of the targeted tumor. There was no change in baseline liver function after ablation. 
Operators may choose to treat lesions with MWA over a conceptual benefit in the setting of increased heat sink near major vessels. ${ }^{108}$ Transarterial approaches may be limited in the setting of watershed territories which tend to overlap near the hepatic hilum and paracaval regions.

Tumors in subcapsular location are also at risk for suboptimal outcomes including progression ${ }^{99,109}$ and tract seeding. ${ }^{110}$ Although uncommon, tract seeding occurs approximately from $\leq 1.5 \%^{110,111}$ to $12.5 \%{ }^{112}$ and has been associated with concurrent biopsy of the lesion. ${ }^{110,111}$ The risk of tract seeding may be decreased by utilizing techniques such as "no touch" tangential probe placement, tract cauterization, minimizing probe repositioning, and avoiding biopsy when possible. ${ }^{113}$ Subcapsular tumors can occasionally pose a challenge to transarterial therapies by their tendency to recruit extrahepatic arterial supply and having poor capacitance in their capsular arterial conduit.

Percutaneous ablation of HCC adjacent to the diaphragm and heart also has an increased risk of technical failure and may be accompanied by shoulder pain, transient lung injury, and temporary cardiac and hemodynamic complications. ${ }^{14,115}$ Utilizing adjunctive techniques such as hydrodissection, inducing pneumoperitoneum, gantry angulation, and real-time probe placement with ultrasound can help mitigate complications and safely reach these hazardous locations.

Many times, a patient will be allocated to a given approach which provides the best ability to visualize the lesion during ablation. In the event of inadequate lesion visualization, preablation intra-arterial lipiodol staining, either the lesion or abutting parenchyma creating a "photo negative" of the tumor, fiducial placement, or the intraprocedural addition of a contrast agent can be performed to increase target conspicuity. Image fusion techniques that incorporate separate modalities or generate real-time biologic feedback of ablation and cavity formation are now available and can be useful in select patients, although careful preoperative alignment is critical as this technology may be susceptible to misregistration. ${ }^{116}$

In addition to the aforementioned strategies, TEA and radioembolization are extremely versatile and can be safely used near critical intrahepatic anatomy, subcapsular and other difficult to reach locations, or in transplant patients where tract seeding precludes transplantation. Ablative radioembolization is particularly useful for tumors which may require the ablation of larger margins. While thermal techniques can also achieve large volume ablation, the instantaneous destruction of significant parenchymal volumes may be more prone to complications compared with gradual devitalization over 1 to 3 months via ablative radioembolization.

\section{Extrahepatic Anatomy}

The presence of critical extrahepatic anatomy in close proximity to a thermal ablation target, such as the kidney, bowel, diaphragm, lung, and gallbladder, can be managed with displacement techniques such as hydrodissection with saline, dextrose when colligative property benefits are required, carbon dioxide, balloons or stiff wires, and blunt tip needles. When using general anesthesia, alteration of lung volumes can be of benefit. Repositioning the patient either decubitus or prone may occasionally provide adequate internal rearrangement to permit ablation. The operator should also modulate the power of each probe as needed and aim the tip of the probe in the direction they want to minimize energy deposition. Although the presence of native ascites is often drained prior to intervention, it has not demonstrated an increased risk of complication after RFA. ${ }^{117}$

When a critical structure cannot be sufficiently displaced, the use of thermocouples can serve as an intraoperative surveillance. The risk to extrahepatic anatomy can also be minimized by combining modalities and techniques such as MWA and PEI. ${ }^{87}$ Radioembolization can be performed near bowel and bile ducts with reduced risk over thermal ablation but requires adequate conduit. ${ }^{118}$ Additional assistance can be provided laparoscopically or if there is safe approach for external beam radiation therapy.

\section{Individualizing Ablation Approaches}

A thoughtful approach to patient selection for ablation is critical in optimizing outcomes. Treatment intent (i.e., neoadjuvant, definitive, or palliative) is essential in determining risk tolerance and the optimal therapeutic approach. The decision to offer therapy is made in the context of the anticipated life expectancy pending a successful result. For example, the chosen approach for a patient with a MELD score of 20 will vary based on whether they are candidates for transplantation. Likewise, the decision to treat a given tumor stage in a patient with limited comorbidities may be entirely different when applied to a patient with severe chronic systemic illness.

Parenchymal preservation as a function of underlying hepatic substrate must be considered when an ablation modality is chosen. For example, a patient who has recurrence after a right trisegmentectomy, where the hazard of liver failure may be comparable to the risk of an undertreated margin, would benefit from a tissue-sparing approach.

The diverse biologic presentations of HCC have significant influence on treatment approach. Ablation modalities are therefore chosen relative to their ability to generate margin for a given phenotype. For example, an ill-defined 4-cm HCC with non-smooth margins, irregular peritumoral enhancement, a discontinuous capsule, and an AFP above 500 needs to be treated aggressively with a modality that can predictably ablate large volumes such as MWA, radioembolization, or a combination therapy. Alternatively, a lower risk HCC that is well defined, slow growing, and $\leq 3 \mathrm{~cm}$ can be targeted with any thermal-based modality the operator is most experienced with.

The location and architectural makeup of a tumor exerts tremendous influence on treatment approach based on its visualization, proximity to critical structures, the quality of its vascular conduit, and accessibility. A central lesion in a watershed territory may be treated with thermal ablation whereas an exophytic lesion near bowel with a single vascular pedicle may be a good candidate for radioembolization. Similarly, adjacent extrahepatic structures (such the stomach, bowel, gallbladder, heart, lung, diaphragm, and kidney) exert equal influence on treatment approach. A $1.5-\mathrm{cm}$ segment three HCC herniating into the stomach would 


\begin{tabular}{|c|c|c|c|c|c|c|c|c|c|c|}
\hline \multirow[t]{2}{*}{ Modality } & \multicolumn{2}{|c|}{ Size } & \multicolumn{2}{|c|}{ Biology } & \multicolumn{2}{|c|}{ Anatomy } & \multicolumn{2}{|c|}{ Vascular Conduit } & \multicolumn{2}{|c|}{ Liver Volume } \\
\hline & $\leq 3 \mathrm{~cm}$ & $>3 \mathrm{~cm}$ & Fovorable & Aggressive & Favorable & Challenging & Adequate & Poor & Expendabie & Not Expendabie \\
\hline RFA & & & & & & & $\mathrm{N} / \mathrm{A}$ & $N / A$ & & \\
\hline MWA & & & & & & & $\mathrm{N} / \mathrm{A}$ & $N / A$ & & \\
\hline Cryoablation & & & & & & & $N / A$ & $N / A$ & & \\
\hline IRE & & & & & & & $\mathrm{N} / \mathrm{A}$ & $\mathrm{N} / \mathrm{A}$ & & \\
\hline PEI & & & & & & & $\mathrm{N} / \mathrm{A}$ & $\mathrm{N} / \mathrm{A}$ & & \\
\hline TEA & & & & & & & & & & \\
\hline RE & & & & & & & & & & \\
\hline SBRT & & & & & & & $N / A$ & $\mathrm{~N} / \mathrm{A}$ & & \\
\hline
\end{tabular}

\begin{tabular}{|l|l|}
\hline & Generally Recommended \\
\hline & Experienced Operators \\
\hline & Consider Alternative \\
\hline & Generally Not Recommended \\
\hline
\end{tabular}

Fig. 5 Example score card for ablation modality considerations in the context of hepatocellular carcinoma size, biology, intra- and extrahepatic anatomy, vascular conduit, and liver volume. These guidelines are meant to stimulate thoughtful ablation, but operators should use techniques with which they are most experienced in accordance with institutional practice. Green: generally recommended; blue: experienced operators; yellow: consider alternative; red: generally not recommended.

require adjunctive displacement for most thermal modalities but may be easily treated with PEI.

Although a well-rounded and versatile interventional oncology program is best, institutional and operator experience can greatly affect the success of any given locoregional therapy. ${ }^{99,119,120}$ There is also emerging data to support that centers with expertise in more therapeutic modalities are associated with improved outcomes for patients with HCC. ${ }^{121}$

\section{Conclusion}

Ablative therapy for HCC is now a mature treatment that is regarded as standard of care in selected settings. Judicious patient selection and comprehensive assessment of tumor presentation are indispensable to successful curative outcomes (-Fig. 5). The strategies proposed in this article provide an approach to high-quality, individualized, HCC ablation.

\section{Conflict of Interest}

Beau Toskich, MD is an advisor for BTG, Johnson \& Johnson, Boston Scientific, and AstraZeneca.t

\section{References}

1 Marrero JA, Kulik LM, Sirlin CB, et al. Diagnosis, staging, and management of hepatocellular carcinoma: 2018 practice guidance by the American Association for the Study of Liver Diseases. Hepatology 2018;68(02):723-750

2 Galle PR, Forner A, Llovet JM, et al; European Association for the Study of the Liver. Electronic Address: easloffice@easloffice.eu; European Association for the Study of the Liver. EASL Clinical
Practice Guidelines: management of hepatocellular carcinoma. J Hepatol 2018;69(01):182-236

3 Llovet JM, Fuster J, Bruix J; Barcelona-Clínic Liver Cancer Group. The Barcelona approach: diagnosis, staging, and treatment of hepatocellular carcinoma. Liver Transpl 2004;10(02, Suppl 1): S115-S120

4 Feng K, Yan J, Li X, et al. A randomized controlled trial of radiofrequency ablation and surgical resection in the treatment of small hepatocellular carcinoma. JHepatol 2012;57(04): 794-802

5 Chen MS, Li JQ, Zheng Y, et al. A prospective randomized trial comparing percutaneous local ablative therapy and partial hepatectomy for small hepatocellular carcinoma. Ann Surg 2006; 243(03):321-328

6 Uhlig J, Sellers CM, Stein SM, Kim HS. Radiofrequency ablation versus surgical resection of hepatocellular carcinoma: contemporary treatment trends and outcomes from the United States National Cancer Database. Eur Radiol 2019;29(05):2679-2689

7 Cucchetti A, Piscaglia F, Cescon M, et al. Cost-effectiveness of hepatic resection versus percutaneous radiofrequency ablation for early hepatocellular carcinoma. J Hepatol 2013;59(02):300-307

8 He Q Jiang JJ, Jiang YX, Wang WT, Yang L; Liver Surgery Group. Health-related quality of life comparisons after radical therapy for early-stage hepatocellular carcinoma. Transplant Proc 2018; 50(05):1470-1474

9 Llovet JM, Schwartz M, Mazzaferro V. Resection and liver transplantation for hepatocellular carcinoma. Semin Liver Dis 2005; 25(02):181-200

10 European Association for the Study of the Liver; European Organisation for Research and Treatment of Cancer. EASL-EORTC clinical practice guidelines: management of hepatocellular carcinoma. J Hepatol 2012;56(04):908-943

11 Llovet JM, Fuster J, Bruix J. Intention-to-treat analysis of surgical treatment for early hepatocellular carcinoma: resection versus transplantation. Hepatology 1999;30(06):1434-1440 
12 Chagas AL, Felga GEG, Diniz MA, et al; Brazilian HCC Study Group. Hepatocellular carcinoma recurrence after liver transplantation in a Brazilian multicenter study: clinical profile and prognostic factors of survival. Eur J Gastroenterol Hepatol 2019;31(09):1148-1156

13 Hsu CY, Huang YH, Chiou YY, et al. Comparison of radiofrequency ablation and transarterial chemoembolization for hepatocellular carcinoma within the Milan criteria: a propensity score analysis. Liver Transpl 2011;17(05):556-566

14 Shen PC, Chang WC, Lo CH, et al. Comparison of stereotactic body radiation therapy and transarterial chemoembolization for unresectable medium-sized hepatocellular carcinoma. Int J Radiat Oncol Biol Phys 2019;105(02):307-318

15 Vouche M, Habib A, Ward TJ, et al. Unresectable solitary hepatocellular carcinoma not amenable to radiofrequency ablation: multicenter radiology-pathology correlation and survival of radiation segmentectomy. Hepatology 2014;60(01):192-201

$16 \mathrm{Ni} \mathrm{JY}$, Sun HL, Chen YT, et al. Prognostic factors for survival after transarterial chemoembolization combined with microwave ablation for hepatocellular carcinoma. World J Gastroenterol 2014;20(46):17483-17490

17 Oken MM, Creech RH, Tormey DC, et al. Toxicity and response criteria of the Eastern Cooperative Oncology Group. Am J Clin Oncol 1982;5(06):649-655

18 National Comprehensive Cancer Network. Older Adult Oncology (Version 1.2019). Available at: https://www.nccn.org/professionals/physician_gls/pdf/senior.pdf. Accessed September 1, 2019

19 Lee SK, Song MJ, Kim SH, Park M. Comparing various scoring system for predicting overall survival according to treatment modalities in hepatocellular carcinoma focused on plateletalbumin-bilirubin (PALBI) and albumin-bilirubin (ALBI) grade: a nationwide cohort study. PLoS One 2019;14(05):e0216173

20 Liu PH, Hsu CY, Hsia CY, et al. ALBI and PALBI grade predict survival for HCC across treatment modalities and BCLC stages in the MELD Era. J Gastroenterol Hepatol 2017;32(04):879-886

21 Antkowiak M, Gabr A, Das A, et al. Prognostic role of albumin, bilirubin, and ALBI scores: analysis of 1000 patients with hepatocellular carcinoma undergoing radioembolization. Cancers (Basel) 2019;11(06):E879

22 Sarkar J, DeLeon T, Wong LL. MELD score and AST-to-platelet ratio index (APRI) predict long-term survival in patients with a small hepatocellular carcinoma following non-transplant therapies: a pilot study. Hepatoma Res 2017;3:79-85

23 Cho JY, Choi MS, Lee GS, et al. Clinical significance and predictive factors of early massive recurrence after radiofrequency ablation in patients with a single small hepatocellular carcinoma. Clin Mol Hepatol 2016;22(04):477-486

$24 \mathrm{Kim}$ CG, Lee HW, Choi HJ, et al. Development and validation of a prognostic model for patients with hepatocellular carcinoma undergoing radiofrequency ablation. Cancer Med 2019;8(11): 5023-5032

25 Su XF, Li N, Chen XF, Zhang L, Yan M. Incidence and risk factors for liver abscess after thermal ablation of liver neoplasm. Hepat Mon 2016;16(07):e34588

26 Odisio BC, Richter M, Aloia TA, et al. Use of prophylactic antibiotics to prevent abscess formation following hepatic ablation in patients with prior enterobiliary manipulation. J Gastrointest Surg 2016;20(08):1428-1434

27 Chau GY, Lui WY, Tsay SH, et al. Prognostic significance of surgical margin in hepatocellular carcinoma resection: an analysis of 165 Childs' A patients. J Surg Oncol 1997;66(02):122-126

28 Yoshida Y, Kanematsu T, Matsumata T, Takenaka K, Sugimachi K. Surgical margin and recurrence after resection of hepatocellular carcinoma in patients with cirrhosis. Further evaluation of limited hepatic resection. Ann Surg 1989;209(03):297-301

29 Kim YS, Lee WJ, Rhim H, Lim HK, Choi D, Lee JY. The minimal ablative margin of radiofrequency ablation of hepatocellular carcinoma ( $>2$ and $<5 \mathrm{~cm}$ ) needed to prevent local tumor progression: 3D quantitative assessment using CT image fusion. AJR Am J Roentgenol 2010;195(03):758-765

30 Teng $\mathrm{W}$, Liu $\mathrm{KW}$, Lin CC, et al. Insufficient ablative margin determined by early computed tomography may predict the recurrence of hepatocellular carcinoma after radiofrequency ablation. Liver Cancer 2015;4(01):26-38

31 Kim YS, Rhim H, Cho OK, Koh BH, Kim Y. Intrahepatic recurrence after percutaneous radiofrequency ablation of hepatocellular carcinoma: analysis of the pattern and risk factors. Eur J Radiol 2006;59(03):432-441

32 Nakazawa T, Kokubu S, Shibuya A, et al. Radiofrequency ablation of hepatocellular carcinoma: correlation between local tumor progression after ablation and ablative margin. AJR Am J Roentgenol 2007;188(02):480-488

33 Okuwaki Y, Nakazawa T, Shibuya A, et al. Intrahepatic distant recurrence after radiofrequency ablation for a single small hepatocellular carcinoma: risk factors and patterns. JGastroenterol 2008;43(01):71-78

34 Liao M, Zhong X, Zhang J, et al. Radiofrequency ablation using a $10-\mathrm{mm}$ target margin for small hepatocellular carcinoma in patients with liver cirrhosis: a prospective randomized trial. J Surg Oncol 2017;115(08):971-979

35 Ke S, Ding XM, Qian X-J, et al. Radiofrequency ablation of hepatocellular carcinoma sized $>3$ and $\leq 5 \mathrm{~cm}$ : is ablative margin of more than $1 \mathrm{~cm}$ justified? World J Gastroenterol 2013;19(42):7389-7398

36 Han J, Li ZL, Xing H, et al. The impact of resection margin and microvascular invasion on long-term prognosis after curative resection of hepatocellular carcinoma: a multi-institutional study. HPB (Oxford) 2019;21(08):962-971

37 Cuccurullo V, Di Stasio GD, Mazzarella G, Cascini GL. Microvascular invasion in HCC: the molecular imaging perspective. Contrast Media Mol Imaging 2018;2018:9487938

38 Wang X, Deng Q Feng K, et al. Insufficient radiofrequency ablation promotes hepatocellular carcinoma cell progression via autophagy and the CD133 feedback loop. Oncol Rep 2018; 40(01):241-251

39 Xu M, Doyle MM, Banan B, et al. Neoadjuvant locoregional therapy and recurrent hepatocellular carcinoma after liver transplantation. JAm Coll Surg 2017;225(01):28-40

40 Zhang R, Ma M, Lin XH, et al. Extracellular matrix collagen I promotes the tumor progression of residual hepatocellular carcinoma after heat treatment. BMC Cancer 2018;18(01):901

41 Yoshida S, Kornek M, Ikenaga N, et al. Sublethal heat treatment promotes epithelial-mesenchymal transition and enhances the malignant potential of hepatocellular carcinoma. Hepatology 2013;58(05):1667-1680

42 Tan L, Chen S, Wei G, et al. Sublethal heat treatment of hepatocellular carcinoma promotes intrahepatic metastasis and stemness in a VEGFR1-dependent manner. Cancer Lett 2019; 460:29-40

43 Agopian VG, Harlander-Locke MP, Ruiz RM, et al. Impact of pretransplant bridging locoregional therapy for patients with hepatocellular carcinoma within Milan criteria undergoing liver transplantation: analysis of 3601 patients from the US Multicenter HCC Transplant Consortium. Ann Surg 2017;266(03):525-535

44 Calderaro J, Ziol M, Paradis V, Zucman-Rossi J. Molecular and histological correlations in liver cancer. JHepatol 2019;71(03): 616-630

45 Taouli B, Hoshida Y, Kakite S, et al. Imaging-based surrogate markers of transcriptome subclasses and signatures in hepatocellular carcinoma: preliminary results. Eur Radiol 2017;27(11): 4472-4481

46 Yoneda N, Matsui O, Kobayashi S, et al. Current status of imaging biomarkers predicting the biological nature of hepatocellular carcinoma. Jpn J Radiol 2019;37(03):191-208

47 Rhee H, An C, Kim HY, Yoo JE, Park YN, Kim MJ. Hepatocellular carcinoma with irregular rim-like arterial phase 
hyperenhancement: more aggressive pathologic features. Liver Cancer 2019;8(01):24-40

48 Xu P, Zeng M, Liu K, Shan Y, Xu C, Lin J. Microvascular invasion in small hepatocellular carcinoma: is it predictable with preoperative diffusion-weighted imaging? J Gastroenterol Hepatol 2014; 29(02):330-336

49 Ryu T, Takami Y, Wada Y, et al. A clinical scoring system for predicting microvascular invasion in patients with hepatocellular carcinoma within the Milan criteria. J Gastrointest Surg 2019; 23(04):779-787

50 Navadgi S, Chang CC, Bartlett A, McCall J, Pandanaboyana S. Systematic review and meta-analysis of outcomes after liver resection in patients with hepatocellular carcinoma (HCC) with and without bile duct thrombus. HPB (Oxford) 2016;18(04):312-316

51 Shin WY, Suh KS, Lee HW, et al. Prognostic factors affecting survival after recurrence in adult living donor liver transplantation for hepatocellular carcinoma. Liver Transpl 2010;16(05):678-684

52 Cha C, Fong Y, Jarnagin WR, Blumgart LH, DeMatteo RP. Predictors and patterns of recurrence after resection of hepatocellular carcinoma. J Am Coll Surg 2003;197(05):753-7583

$53 \mathrm{Kim}$ JK, Kim HD, Jun MJ, et al. Tumor volume doubling time as a dynamic prognostic marker for patients with hepatocellular carcinoma. [Erratum appears in Dig Dis Sci. 2017 Nov;62 (11):3259] Dig Dis Sci 2017;62(10):2923-2931

54 Min JH, Kim YK, Lim S, Jeong WK, Choi D, Lee WJ. Prediction of microvascular invasion of hepatocellular carcinomas with gadoxetic acid-enhanced MR imaging: Impact of intra-tumoral fat detected on chemical-shift images. Eur J Radiol 2015;84(06): 1036-1043

55 Ma X, Wei J, Gu D, et al. Preoperative radiomics nomogram for microvascular invasion prediction in hepatocellular carcinoma using contrast-enhanced CT. Eur Radiol 2019;29(07):3595-3605

56 Ahn SJ, Kim JH, Park SJ, Kim ST, Han JK. Hepatocellular carcinoma: preoperative gadoxetic acid-enhanced MR imaging can predict early recurrence after curative resection using image features and texture analysis. Abdom Radiol (NY) 2019;44(02): 539-548

57 Lok AS, Sterling RK, Everhart JE, et al; HALT-C Trial Group. Des- $\gamma$ carboxy prothrombin and $\alpha$-fetoprotein as biomarkers for the early detection of hepatocellular carcinoma. Gastroenterology 2010;138(02):493-502

58 Paul SB, Gulati MS, Sreenivas V, et al. Evaluating patients with cirrhosis for hepatocellular carcinoma: value of clinical symptomatology, imaging and alpha-fetoprotein. Oncology 2007;72 (Suppl 1):117-123

59 Gurakar A, Ma M, Garonzik-Wang J, et al. Clinicopathological distinction of low-AFP-secreting vs. high-AFP-secreting hepatocellular carcinomas. Ann Hepatol 2018;17(06):1052-1066

60 Hameed B, Mehta N, Sapisochin G, Roberts JP, Yao FY. Alphafetoprotein level $>1000 \mathrm{ng} / \mathrm{mL}$ as an exclusion criterion for liver transplantation in patients with hepatocellular carcinoma meeting the Milan criteria. Liver Transpl 2014;20(08):945-951

61 Li R, Yang D, Tang CL, et al. Combined hepatocellular carcinoma and cholangiocarcinoma (biphenotypic) tumors: clinical characteristics, imaging features of contrast-enhanced ultrasound and computed tomography. BMC Cancer 2016;16:158

62 Lee JH, Chung GE, Yu SJ, et al. Long-term prognosis of combined hepatocellular and cholangiocarcinoma after curative resection comparison with hepatocellular carcinoma and cholangiocarcinoma. JClin Gastroenterol 2011;45(01):69-75

63 Lee $\mathrm{CH}$, Hsieh SY, Chang CJ, Lin YJ. Comparison of clinical characteristics of combined hepatocellular-cholangiocarcinoma and other primary liver cancers. J Gastroenterol Hepatol 2013;28 (01):122-127

64 Chen K, Zhan MX, Hu BS, et al. Combination of the neutrophil to lymphocyte ratio and the platelet to lymphocyte ratio as a useful predictor for recurrence following radiofrequency ablation of hepatocellular carcinoma. Oncol Lett 2018;15(01):315-323
65 Lee S, Rhim H, Kim YS, Kang TW, Song KD. Post-ablation desgamma-carboxy prothrombin level predicts prognosis in hepatitis B-related hepatocellular carcinoma. Liver Int 2016;36(04): 580-587

66 Xia F, Lai ECH, Lau WY, et al. High serum hyaluronic acid and HBV viral load are main prognostic factors of local recurrence after complete radiofrequency ablation of hepatitis B-related small hepatocellular carcinoma. Ann Surg Oncol 2012;19(04):1284-1291

67 Lou J, Zhang L, Lv S, Zhang C, Jiang S. Biomarkers for hepatocellular carcinoma. Biomarkers in Cancer 2017;9:1-9

68 Berretta M, Cavaliere C, Alessandrini L, et al. Serum and tissue markers in hepatocellular carcinoma and cholangiocarcinoma: clinical and prognostic implications. Oncotarget 2017;8(08): 14192-14220

69 Lu DSK, Yu NC, Raman SS, et al. Radiofrequency ablation of hepatocellular carcinoma: treatment success as defined by histologic examination of the explanted liver. Radiology 2005; 234(03):954-960

70 Mazzaferro V, Battiston C, Perrone S, et al. Radiofrequency ablation of small hepatocellular carcinoma in cirrhotic patients awaiting liver transplantation: a prospective study. Ann Surg 2004;240(05):900-909

71 Facciorusso A, Di Maso M, Muscatiello N. Microwave ablation versus radiofrequency ablation for the treatment of hepatocellular carcinoma: a systematic review and meta-analysis. Int $\mathrm{J}$ Hyperthermia 2016;32(03):339-344

72 Kamal A, Elmoety AAA, Rostom YAM, Shater MS, Lashen SA. Percutaneous radiofrequency versus microwave ablation for management of hepatocellular carcinoma: a randomized controlled trial. J Gastrointest Oncol 2019;10(03):562-571

73 Weber SM, Lee FT Jr, Warner TF, Chosy SG, Mahvi DM. Hepatic cryoablation: US monitoring of extent of necrosis in normal pig liver. Radiology 1998;207(01):73-77

74 Wang C, Wang H, Yang W, et al. Multicenter randomized controlled trial of percutaneous cryoablation versus radiofrequency ablation in hepatocellular carcinoma. Hepatology 2015; 61(05):1579-1590

75 Rong G, Bai W, Dong Z, et al. Long-term outcomes of percutaneous cryoablation for patients with hepatocellular carcinoma within Milan criteria. PLoS One 2015;10(04):e0123065

76 Di Costanzo GG, Tortora R, D’Adamo G, et al. Radiofrequency ablation versus laser ablation for the treatment of small hepatocellular carcinoma in cirrhosis: a randomized trial. JGastroenterol Hepatol 2015;30(03):559-565

77 Sutter O, Calvo J, N'Kontchou G, et al. Safety and efficacy of irreversible electroporation for the treatment of hepatocellular carcinoma not amenable to thermal ablation techniques: a retrospective single-center case series. Radiology 2017;284 (03):877-886

78 Cheng RG, Bhattacharya R, Yeh MM, Padia SA. Irreversible electroporation can effectively ablate hepatocellular carcinoma to complete pathologic necrosis. JVasc Interv Radiol 2015;26 (08):1184-1188

79 Luo W, Zhang Y, He G, et al. Effects of radiofrequency ablation versus other ablating techniques on hepatocellular carcinomas: a systematic review and meta-analysis. World J Surg Oncol 2017; 15(01):126

80 Facciorusso A, Serviddio G, Muscatiello N. Local ablative treatments for hepatocellular carcinoma: an updated review. World J Gastrointest Pharmacol Ther 2016;7(04):477-489

81 Wang S, Zhuang L, Meng Z. Hepatocellular carcinoma more than $3 \mathrm{~cm}$ in diameter: a systematic review of transcatheter arterial chemoembolization plus percutaneous ethanol injection versus transcatheter arterial chemoembolization alone. ISRN Gastroenterol 2013;2013:526024

82 Pompili M, Mirante VG, Rondinara G, et al. Percutaneous ablation procedures in cirrhotic patients with hepatocellular carcinoma submitted to liver transplantation: Assessment of efficacy at 
explant analysis and of safety for tumor recurrence. Liver Transpl 2005;11(09):1117-1126

83 Branco F, Brú C, Vilana R, Bianchi L, Alves de Mattos A. Percutaneous ethanol injection before liver transplantation in the hepatocellular carcinoma. Ann Hepatol 2009;8(03):220-227

$84 \mathrm{Yu}$ SCH, Hui EP, Tang P, et al. Transarterial ethanol ablation for unresectable hepatocellular carcinoma: analysis of clinical and tumor outcomes. JVasc Interv Radiol 2016;27(05):639-649

$85 \mathrm{Yu} \mathrm{SCH}$, Hui JWY, Hui EP, et al. Unresectable hepatocellular carcinoma: randomized controlled trial of transarterial ethanol ablation versus transcatheter arterial chemoembolization. Radiology 2014;270(02):607-620

86 Fu Y, Zhao X, Yun $\mathrm{Q}$ et al. Transarterial chemoembolization (TACE) plus percutaneous ethanol injection (PEI) for the treatment of unresectable hepatocellular carcinoma: a meta-analysis of randomized controlled trials. Int J Clin Exp Med 2015;8(07): 10388-10400

87 Huang H, Liang P, Yu XL, et al. Safety assessment and therapeutic efficacy of percutaneous microwave ablation therapy combined with percutaneous ethanol injection for hepatocellular carcinoma adjacent to the gallbladder. Int J Hyperthermia 2015;31(01): 40-47

88 Ahmed AF, Samreen N, Grajo JR, et al. Angiosomal radiopathologic analysis of transarterial radioembolization for the treatment of hepatocellular carcinoma. Abdom Radiol (NY) 2018;43 (07):1825-1836

89 Lewandowski RJ, Gabr A, Abouchaleh N, et al. Radiation segmentectomy: potential curative therapy for early hepatocellular carcinoma. Radiology 2018;287(03):1050-1058

90 Shah JL, Zendejas-Ruiz IR, Thornton LM, et al. Neoadjuvant transarterial radiation lobectomy for colorectal hepatic metastases: a small cohort analysis on safety, efficacy, and radiopathologic correlation. J Gastrointest Oncol 2017;8 (03):E43-E51

91 Gates VL, Hickey R, Marshall K, et al. Gastric injury from (90)Y to left hepatic lobe tumors adjacent to the stomach: fact or fiction? Eur J Nucl Med Mol Imaging 2015;42(13):2038-2044

92 Meek J, Fletcher S, Gauss CH, Bezold S, Borja-Cacho D, Meek M. Temporary balloon occlusion for hepatic arterial flow redistribution during yttrium-90 radioembolization. JVasc Interv Radiol 2019;30(08):1201-1206

93 Su TS, Liang P, Lu HZ, et al. Stereotactic body radiation therapy for small primary or recurrent hepatocellular carcinoma in 132 Chinese patients. J Surg Oncol 2016;113(02):181-187

94 Sapisochin G, Barry A, Doherty M, et al. Stereotactic body radiotherapy vs. TACE or RFA as a bridge to transplant in patients with hepatocellular carcinoma. An intention-to-treat analysis. J Hepatol 2017;67(01):92-99

95 Uemura T, Kirichenko A, Bunker M, Vincent M, Machado L, Thai N. Stereotactic body radiation therapy: a new strategy for locoregional treatment for hepatocellular carcinoma while awaiting liver transplantation. World J Surg 2019;43(03):886-893

96 Chan ACY, Cheung TT, Fan ST, et al. Survival analysis of highintensity focused ultrasound therapy versus radiofrequency ablation in the treatment of recurrent hepatocellular carcinoma. Ann Surg 2013;257(04):686-692

97 Cheung TT, Fan ST, Chu FSK, et al. Survival analysis of highintensity focused ultrasound ablation in patients with small hepatocellular carcinoma. HPB (Oxford) 2013;15(08):567-573

98 Lu DSK, Raman SS, Limanond P, et al. Influence of large peritumoral vessels on outcome of radiofrequency ablation of liver tumors. J Vasc Interv Radiol 2003;14(10):1267-1274

99 Mulier S, Ni Y, Jamart J, Ruers T, Marchal G, Michel L. Local recurrence after hepatic radiofrequency coagulation: multivariate meta-analysis and review of contributing factors. Ann Surg 2005;242(02):158-171

100 Kang TW, Lim HK, Lee MW, et al. Aggressive intrasegmental recurrence of hepatocellular carcinoma after radiofrequency ablation: risk factors and clinical significance. Radiology 2015; 276(01):274-285

101 Teratani T, Yoshida H, Shiina S, et al. Radiofrequency ablation for hepatocellular carcinoma in so-called high-risk locations. Hepatology 2006;43(05):1101-1108

102 Kondo Y, Shiina S, Tateishi R, et al. Intrahepatic bile duct dilatation after percutaneous radiofrequency ablation for hepatocellular carcinoma: impact on patient's prognosis. Liver Int 2011;31(02):197-205

$103 \mathrm{Li} \mathrm{X}$, Yu J, Liang P, et al. Ultrasound-guided percutaneous microwave ablation assisted by three-dimensional visualization operative treatment planning system and percutaneous transhepatic cholangial drainage with intraductal chilled saline perfusion for larger hepatic hilum hepatocellular $(\mathrm{D} \geq 3 \mathrm{~cm})$ : preliminary results. Oncotarget 2017;8(45):79742-79749

104 Silk MT, Wimmer T, Lee KS, et al. Percutaneous ablation of peribiliary tumors with irreversible electroporation. JVasc Interv Radiol 2014;25(01):112-118

105 Dollinger M, Zeman F, Niessen C, et al. Bile duct injury after irreversible electroporation of hepatic malignancies: evaluation of MR imaging findings and laboratory values. JVasc Interv Radiol 2016;27(01):96-103

106 Bhutiani N, Philips P, Scoggins CR, McMasters KM, Potts MH, Martin RCG. Evaluation of tolerability and efficacy of irreversible electroporation (IRE) in treatment of Child-Pugh B (7/8) hepatocellular carcinoma (HCC). HPB (Oxford) 2016;18(07):593-599

107 Littrup PJ, Aoun HD, Adam B, Krycia M, Prus M, Shields A. Percutaneous cryoablation of hepatic tumors: long-term experience of a large U.S. series. Abdom Radiol (NY) 2016;41(04): 767-780

108 Dou JP, Yu J, Yang XH, et al. Outcomes of microwave ablation for hepatocellular carcinoma adjacent to large vessels: a propensity score analysis. Oncotarget 2017;8(17):28758-28768

109 Lai ZC, Liang JY, Chen LD, et al. Do hepatocellular carcinomas located in subcapsular space or in proximity to vessels increase the rate of local tumor progression? A meta-analysis. Life Sci 2018;207:381-385

110 Francica G, Meloni MF, de Sio I, et al. Radiofrequency and microwave ablation of subcapsular hepatocellular carcinoma accessed by direct puncture: safety and efficacy. Eur J Radiol 2016;85(04):739-743

111 Yu J, Liang P, Yu XL, Cheng ZG, Han ZY, Dong BW. Needle track seeding after percutaneous microwave ablation of malignant liver tumors under ultrasound guidance: analysis of 14-year experience with 1462 patients at a single center. Eur J Radiol 2012;81(10):2495-2499

112 Llovet JM, Vilana R, Brú C, et al; Barcelona Clínic Liver Cancer (BCLC) Group. Increased risk of tumor seeding after percutaneous radiofrequency ablation for single hepatocellular carcinoma. Hepatology 2001;33(05):1124-1129

113 Patel PA, Ingram L, Wilson IDC, Breen DJ. No-touch wedge ablation technique of microwave ablation for the treatment of subcapsular tumors in the liver. J Vasc Interv Radiol 2013;24(08): 1257-1262

114 Cha DI, Kang TW, Song KD, et al. Radiofrequency ablation for subcardiac hepatocellular carcinoma: therapeutic outcomes and risk factors for technical failure. Eur Radiol 2019;29(05): $2706-2715$

115 Kang TW, Rhim H, Kim EY, et al. Percutaneous radiofrequency ablation for the hepatocellular carcinoma abutting the diaphragm: assessment of safety and therapeutic efficacy. Korean J Radiol 2009;10(01):34-42

116 Calandri M, Mauri G, Yevich S, et al. Fusion imaging and virtual navigation to guide percutaneous thermal ablation of hepatocellular carcinoma: a review of the literature. Cardiovasc Intervent Radiol 2019;42(05):639-647

117 Cha J, Rhim H, Lee JY, et al. Percutaneous radiofrequency ablation of hepatocellular carcinoma: assessment of safety in 
patients with ascites. AJR Am J Roentgenol 2009;193(05): W424-W429

118 Salem R, Padia SA, Lam M, et al. Clinical and dosimetric considerations for Y90: recommendations from an international multidisciplinary working group. Eur J Nucl Med Mol Imaging 2019;46(08):1695-1704

119 Hildebrand P, Leibecke T, Kleemann M, et al. Influence of operator experience in radiofrequency ablation of malignant liver tumours on treatment outcome. Eur J Surg Oncol 2006;32(04): 430-434
120 Poon RT, Ng KK, Lam CM, et al. Learning curve for radiofrequency ablation of liver tumors: prospective analysis of initial 100 patients in a tertiary institution. Ann Surg 2004;239(04):441-449

121 Jiang JM, Ohri N, Tang J, et al. Centers with more therapeutic modalities are associated with improved outcomes for patients with hepatocellular carcinoma. J Gastrointest Oncol 2019;10 (03):546-553 